\title{
Increased Calcitonin Gene-Related Peptide
}

\section{Expression in DRG and Nerve Fibers Proliferation Caused by Nonunion Fracture in Rats}

\author{
Yusuke Kasai \\ Koji Aso \\ Masashi Izumi $\mathbb{D}$ \\ Hiroyuki Wada \\ Junpei Dan \\ Yoshinori Satake \\ Toru Morimoto \\ Masahiko Ikeuchi \\ Department of Orthopedic Surgery, \\ Kochi Medical School, Kochi University, \\ Nankoku, 783-8505, Japan
}

Correspondence: Koji Aso

Department of Orthopedic Surgery,

Kochi Medical School, Kochi University,

I85-I Oko-cho Kohasu, Nankoku, 783-

8505, Japan

Tel $+8 \mathrm{I}-88-880-2386$

$\mathrm{Fax}+8 \mathrm{I}-88-880-2388$

Email koji.aso@gmail.com
Purpose: Nonunion bone fracture can be a cause of persistent pain, but the pathophysiology remains largely unknown. The objective of this study was to identify how nonunion affect persistent pain after fracture. Specifically, we evaluated the association of neuropeptide change in dorsal root ganglia (DRG) and nerve proliferation at fracture sites with pain.

Methods: Rat union and nonunion fracture models were created. A piece of latex glove was placed at the fracture site to create a nonunion model. At 6 weeks after surgery, bone healing was assessed using radiography. In addition, the presence of calcitonin gene-related peptideimmunoreactive (CGRP-IR) DRG at the level of L3 and anti-growth associated protein 43immunoreactive (GAP43-IR) nerve fibers in the scar tissue between the bone fragments were evaluated. Pain-related behavior was assessed using forced treadmill running.

Results: In radiological images at 6 weeks after surgery, callus formation was formed continuously between bone fragments in the union models. On the one hand, a clear gap was detected between fragments in nonunion models. The percentage of CGRP-IR DRG cells and the density of GAP43-IR nerve fibers in the scar tissue between the bone fragments in nonunion models was significantly higher than that in union models $(p<0.05)$. An increase in inflammatory cell infiltrate was observed in scar tissues in the nonunion models. During forced treadmill running, rats in the union model could run significantly longer than those in the nonunion models. Conclusion: Increased CGRP expression in DRG cells and abnormal nerve proliferation secondary to prolonged inflammation could lead to persistent pain after bone fracture. In clinical practice, early achievement of bone union may minimize the development of persistent pain after fractures.

Keywords: fracture pain, nerve proliferation, nonunion of fractures

\section{Introduction}

Bone fracture pain is a common problem because it is associated with lower functional performance at discharge from the hospital and poor overall functional prognosis. ${ }^{1}$ Furthermore, if nonunion or delayed union occurs after fractures, fracture pain may persist. In order to manage fracture pain caused by nonunion, fixation surgeries and medications such as non-steroidal anti-inflammatory drugs and acetaminophen are generally recommended. Although the mechanism of pain in bone fracture has been well documented in previous studies, ${ }^{2,3}$ in a previous histological analysis of fracture, sprouting nerve fibers labeled with growth associated protein 43 (GAP 43) were observed in the fracture hematoma and periosteum in the early phase after fracture. ${ }^{4}$ However, the pathophysiology of pain due to nonunion or delayed union is largely 
unknown and nerve fibers proliferation at nonunion site of fractures has not been clarified.

The cell bodies of primary sensory neurons innervating bone are located in the dorsal root ganglia (DRG). The DRG cells can be classified into several subpopulations based on nociceptive phenotype markers. One group contains neuropeptides, such as calcitonin gene-related peptide (CGRP) and substance P (SP), whereas the other group lacks peptides and binds isolectin B4 (IB4). ${ }^{5}$ The peptidergic neurons are regulated by nerve growth factor (NGF) and are considered highly sensitive to inflammation. ${ }^{6}$ Previous animal studies showed that the majority of DRG neurons innervating the bone were CGRP-IR, implying that the bone is highly sensitive to nociceptive stimuli and inflammation. ${ }^{7,8}$ However, nociceptive phenotype alteration of DRG neurons innervating nonunion bone after fractures has not been clarified.

Animal models are useful for elucidating the pain mechanisms in bone fractures with nonunion. In previous studies, nonunion animal models were created by generating segmental bone defects, ${ }^{9}$ periosteal disruption, ${ }^{10}$ and using barriers between fragments. ${ }^{11}$ In this study, a piece of latex glove was placed at the fracture site to create nonunion after bone fracture.

The objective of this study was to identify how nonunion affect persistent pain after fracture. Specifically, we evaluated the association of CGRP expression changes in DRG neurons innervating femoral bone and nerve proliferation at fracture sites with pain. We hypothesized that increased CGRP expression in DRG cells and nerve fibers proliferation in the scar tissue between bone fragments are associated with pain caused by bone fracture in nonunion rat models.

\section{Methods}

\section{Animals}

Twelve-week-old male Sprague-Dawley rats (average weight $400 \mathrm{~g}, \mathrm{n}=10$ ) were used in this study. They were housed in a temperature-controlled room with a light-dark cycle. All experiments were approved by and performed in accordance with the Animal Care and Use Committee of Kochi University (J-00086).

\section{Surgery}

All rats were anesthetized using isoflurane, and the skin overlying the left lateral femur was shaved, sterilized, and incised longitudinally (approximately $3 \mathrm{~cm}$ in length). The quadriceps was retracted to expose the bone, and a complete transverse incision was made with a bone saw in the middle of the femur under direct visualization. Each fragment was fixed with a $1.2 \mathrm{~mm}$ Kirschner wire like an intramedullary nail. In the proximal fragment, the wire was cannulated in a retrograde manner. The distal fragment was then repositioned by using the intramedullary wire to penetrate both the proximal and distal epiphysis of the femur for achieving stability. Finally, the fascia and skin were sutured.

Two types of fracture models were created (union and nonunion models, $\mathrm{n}=5$ for each group). In union models, bone healing was in normal process. In nonunion models, a piece of latex glove was placed between the bone fragments to inhibit bone healing (Figure 1).

\section{Radiological Examination}

We confirmed bone healing from 1 to 10 weeks after surgery in a preliminary study. In the preliminary results, we found callus formation in radiographs, and good stability between bone fragments was achieved at 6 weeks. Therefore, we used rats at 6 weeks after surgery to assess the degree of bone healing using radiography (Latheta ${ }^{\circledR}$ LCT-200 HITACHI, Tokyo, Japan) in this study. In addition, a $3 \mathrm{~kg}$ load was applied as a bending force on the distal fragment of the resected lower limb using the original device to compare stability in each model.
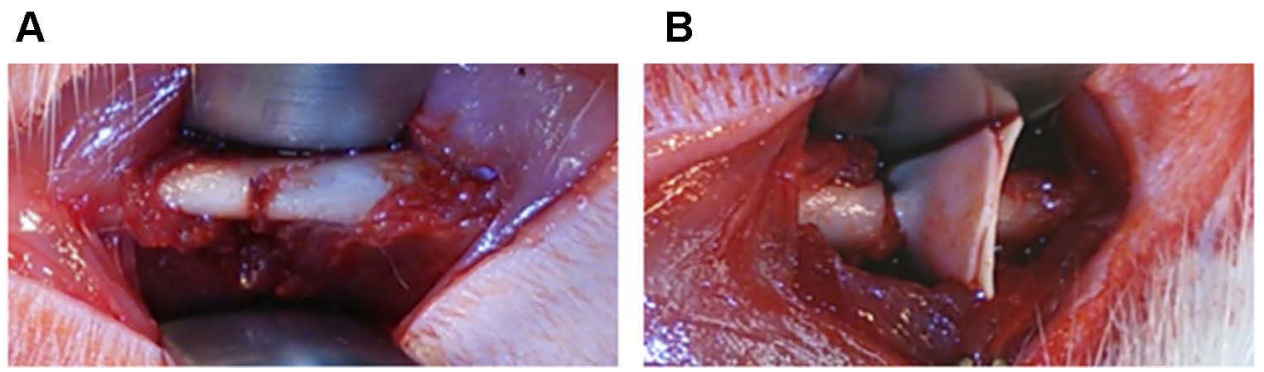

Figure I The intraoperative photographs in the union $(\mathbf{A})$ and nonunion $(\mathbf{B})$ models. A transverse fracture occurred in the middle of the femur in both the models, and a piece of latex glove was inserted between the bone fragments in the nonunion model (B). 


\section{Gait Assessment}

Forced treadmill running was performed to assess gait. The assessment was performed before and at 2, 4, and 6 weeks after surgery. The motor-driven treadmill was designed for rats and operated at a constant speed of $30 \mathrm{~cm} / \mathrm{s}$. It was performed for five min before and at 2 , 4 , and 6 weeks after surgery. The total duration of forced treadmill running (up to a maximum of five min) was measured and used as pain-related behavior.

\section{Histological Examination}

After euthanizing the rats at 6 weeks, the dorsal root ganglias (DRGs) at the level of L3 (left side), and the scar tissue between bone fragments were dissected for immunohistological examination. The DRGs were placed in a $4 \%$ paraformaldehyde in phosphate buffer solution ( $\mathrm{pH} 7.4$ ), soaked in $30 \%$ sucrose, and then rapidly frozen in optical cutting temperature (OCT) compounds to make frozen tissue blocks. All samples were prepared using a cryostat, set at a thickness of 14 $\mu \mathrm{m}$. After washing in the buffer solution three times, the sections were incubated in blocking solution for $1 \mathrm{~h}$ at room temperature. The samples were then washed in the buffer solution three times. Following this, the DRGs were incubated with anti-calcitonin gene-related peptide (CGRP) (1:1000), and the scar tissue was incubated with anti-growth associated protein 43 (GAP43) (1:1000), a marker of axonal growth for 24 hours. After washing the sections three more times in the buffer solution, they were subsequently incubated with goat anti-rabbit IgG antibody conjugated with Alexa Fluor $488(1: 1000)$ at room temperature for $1 \mathrm{~h}$. All sections were washed a final three times in the buffer solution. For the assessment of the DRG, the percentage of CGRP-IR cells in all L3 DRG cells was calculated. DRG cells with visible nuclei were counted in every fifth section to eliminate the possibility of double counting. For the assessment of nerve sprouting in scar tissue between bone fragments, GAP43-IR nerve fibers were manually traced using a light microscope equipped with a computer-aided imaging system, and the length per square area was calculated. GAP43-IR nerve fiber density was determined as the total length of all GAP43-IR nerve fibers in each predetermined area of scar tissue $\left(\mu \mathrm{m} / \mathrm{mm}^{2}\right)$. Nerves were measured in every fifth section. Finally, the scar tissue was stained using the hematoxylin-eosin stain. A Nikon Eclipse 80i microscope (Nikon, Tokyo, Japan) was used for image analysis.

\section{Statistical Analysis}

Statistical analyses were performed using IBM SPSS software (version 26.0). The Kruskal-Wallis and Dunn's tests were used to compare pain-related behavior and immunohistological examination in both groups. Statistical significance was set at $\mathrm{P}<0.05$.

\section{Results}

\section{Radiological Examination}

Representative radiological images are shown in Figure 2. It was confirmed that callus formation was formed continuously between bone fragments in the union models. In nonunion models, calluses appeared, but a clear gap was detected between fragments.

On applying a $3 \mathrm{~kg}$ load of bending force on the distal bone fragment, the gap between the fragments was noted to widen slightly in both groups. However, the gap in the nonunion group tended to be larger than that in the union group (Figure 3).

\section{Gait Assessment}

The total duration of forced treadmill running at 2 weeks was significantly shorter than that before surgery in both groups $(p<0.05)$. At 2 weeks, there was no significant difference

B

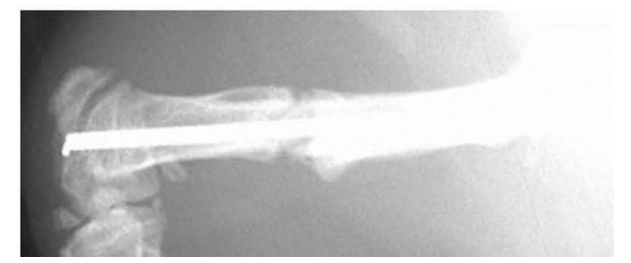

Figure 2 Representative radiological images of the fracture site at 6 weeks after surgery. Callus formation was formed continuously between bone fragments in the union models (A). In the nonunion models (B), a clear gap was detected between the fragments. 
A

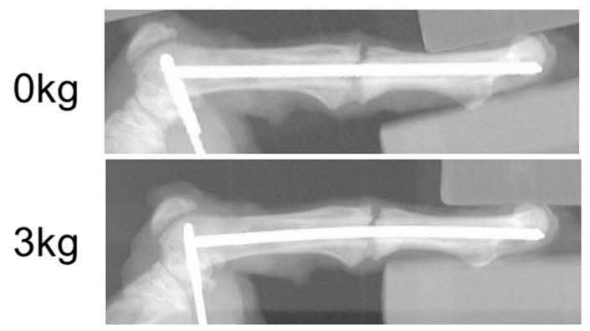

B

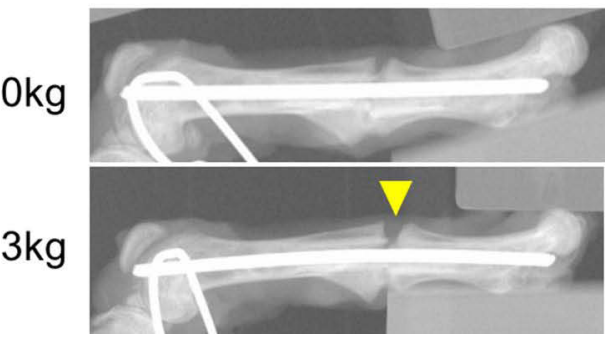

Figure 3 The radiographs comparing the stability between the bone fragments in the union $(\mathbf{A})$ and nonunion models (B) at 6 weeks after surgery. This device could hold the proximal epiphysis and apply a load of $3 \mathrm{~kg}$ as a bending force on the fracture site via the surgical wire of the femoral condyle. The gap in the nonunion group tended to be larger.

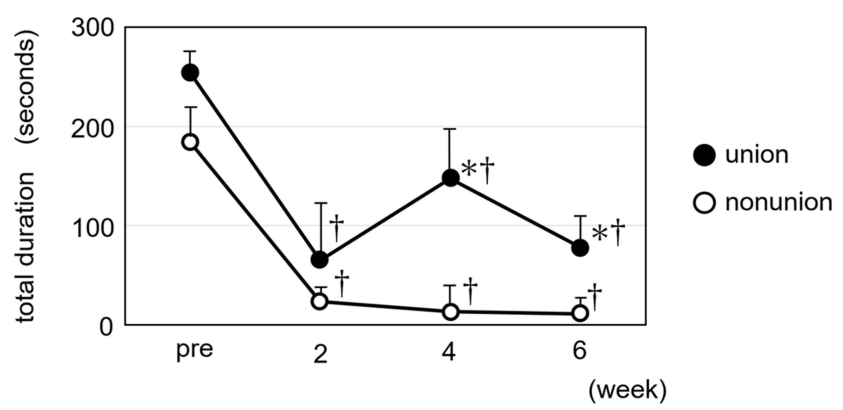

Figure 4 Total duration of forced treadmill running. Data were presented as the mean $\pm \mathrm{SE}, *_{\mathrm{p}}<0.05$ vs nonunion models. The total duration of forced treadmill running in each group was significantly shorter than that before surgery in every period, ${ }^{\dagger} \mathrm{p}<0.05$ vs pre.

between two models. At 4 and 6 weeks, rats in the union model could run significantly longer than those in the nonunion models. Rats in the nonunion models could not run at 6 weeks after surgery $(\mathrm{p}<0.05)$ (Figure 4$)$.

\section{Histological Examination}

Figure 5A-D shows representative photographs of CGRPIR cells in DRGs and Figure 5E shows the percentage of CGRP-IR in the DRG cells. The percentage of CGRP-IR in union models was significantly lower than that of nonunion models.

Figure 6A-D shows representative photographs of the histological analysis of scar tissue between the bone fragments. In the scar tissues of union model rats at 6 weeks after surgery, rich chondrocytes and newly formed woven bone were observed, which is common in standard fracture healing. In contrast, the scar tissues in nonunion model rats were filled with fibroblast-like spindle cells and granulocytes, which represent inflammation. Figure $6 \mathrm{E}$ and F shows representative photographs of GAP43-IR fibers and Figure 6G shows the density of newly sprouting GAP43-IR nerve fibers in the scar tissue. The density of
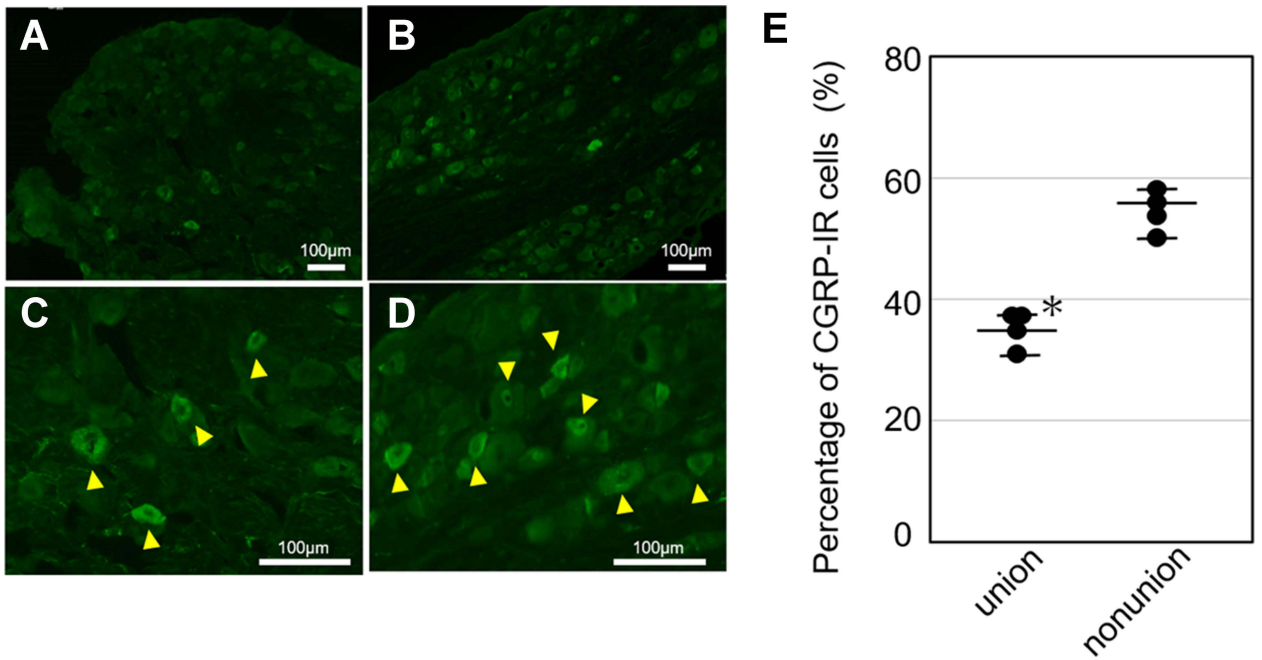

Figure 5 Representative photographs of CGRP-IR DRG cells. (A and $\mathbf{C}$ ) and (B and D) show CGRP-IR DRG cells in the union and nonunion models, respectively. Arrows indicate CGRP-IR cells. (E) shows the percentage of CGRP-IP cells in DRGs. The percentage of CGRP-IR cells in the union models was significantly low compared to the nonunion models. The bar shows the median [interquartile range], ${ }^{*} \mathrm{P}<0.05$ vs nonunion models. 

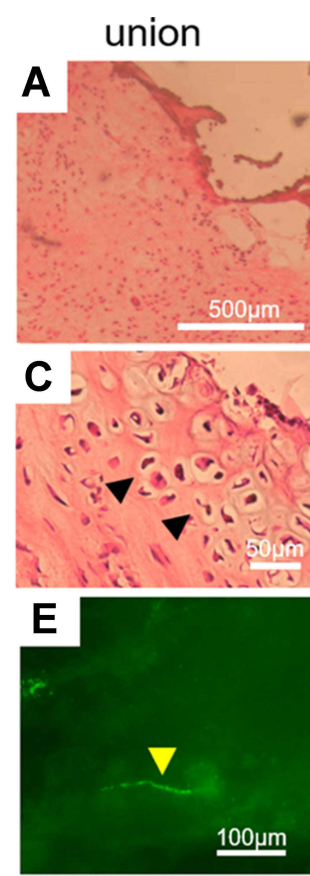

\section{nonunion}
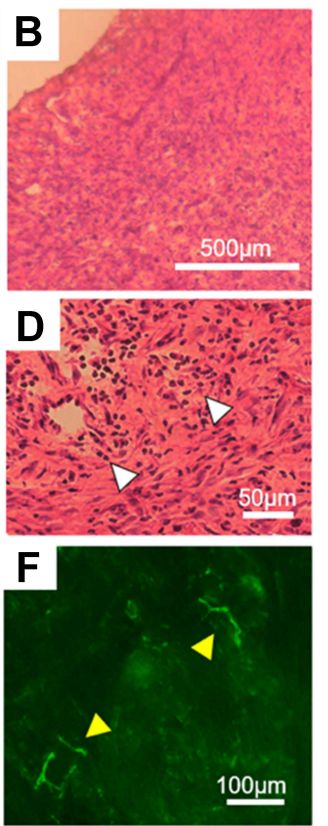

G

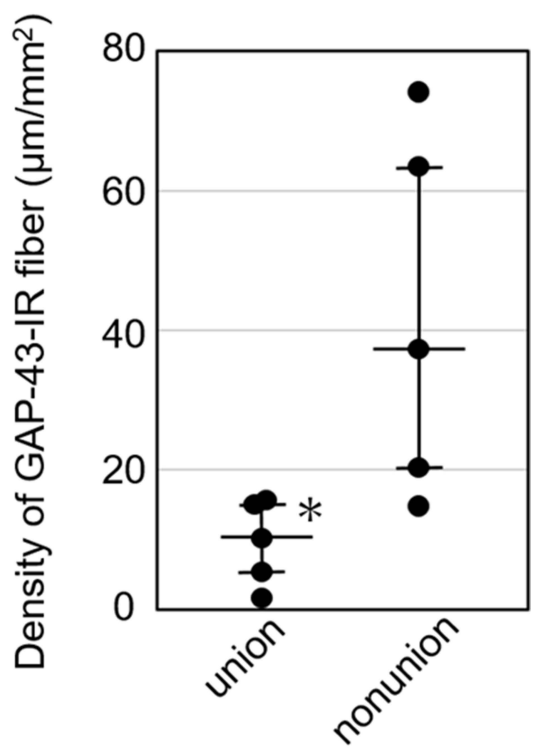

Figure 6 Representative photographs of the scar tissue between the bone fragments. (A-D) shows histological analysis of the scar tissue stained using hematoxylin-eosin in the union ( $\mathbf{A}$ and $\mathbf{C}$ ) and nonunion (B and $\mathbf{D})$ models. In the union models, the tissue consists of rich chondrocytes (black arrow) and newly formed woven bone. The scar tissue of nonunion models is filled with fibroblast-like spindle cells and granulocytes (white arrow). Representative photographs of GAP43-IR fibers in the scar tissue of union (E) and nonunion (F) models. The yellow arrow indicates the GAP43-IR nerve fibers. (G) shows the density of newly sprouting GAP43-IR nerve fibers in the scar tissue. The density of fibers in the scar tissue of union models was lower significantly. The bar shows the median [interquartile range], ${ }^{*} \mathrm{P}<0.05 \mathrm{vs}$ nonunion models.

fibers of union models was significantly lower than that of nonunion models.

\section{Discussion}

To the best of our knowledge, this is the first study to evaluate the association of nonunion in bone fracture with nerve proliferation and pain in rats. The percentage of CGRP-IR in DRG cells innervating femoral bone and the density of GAP43-IR in the nerve fibers of scar tissues between bone fragments was significantly higher than that in union models. Inflammatory cell infiltrates in scar tissue were significantly increased in nonunion models. Thus, increased CGRP expression in DRG cells and abnormal nerve proliferation secondary to inflammatory cell infiltrates in scar tissues could lead to persistent pain after bone fracture. In addition, our nonunion models have can be used for future study for chronic pain after fracture.

Recent study showed pain mechanism after bone fracture. During the inflammatory response to fracture, inflammatory mediators are released and activate and sensitize primary sensory neurons, and intense nerve sprouting. The neural changes in the fracture callus area may be involved in exacerbation of pain. ${ }^{6,12}$ Furthermore, a previous study reported that a high density of sensory nerve fibers and neuroma-like structures were observed to form near the fracture site in nonhealed fractures. In contrast, similar aberrant nerve growth has never been observed in normal femurs. ${ }^{13}$ We extend these findings to identify that nonunion state was associated with increased CGRP expression in DRG cells and abnormal nerve proliferation scar tissues between bone fragments by comparing nonunion with union model. Fracture healing itself is an important factor for pain relief.

The process of fracture healing consists of three phases: inflammation, repair, and remodeling. Fracture healing begins with a local inflammatory response. Factors that affect local inflammation include soft tissue injury, hematoma formation, and fracture stability. ${ }^{14}$ Inflammatory cells are formed in the hematoma of the fracture site, which release inflammatory mediators such as prostaglandins, nerve growth factors, cytokines, and neuropeptides. ${ }^{13}$ Through this process, these mediators promote the growth of new nerve fibers. CGRP, in particular, regulates bone formation and sensory innervation of the bone. ${ }^{15}$ The nerve fibers are well identified in the early phase following fractures which are associated with the initiation of bone formation, and usually regress in the late phase. ${ }^{16}$ However, our results showed that nonunion state 
prolongs the period of increased inflammatory cells in the scar tissue between the bone fragments. Inflammatory cells produce cytokines which promote the growth of nerve fibers. ${ }^{17}$ Similar phenomena were also observed in different animal models and diseases. Intervertebral disc inflammation promotes axonal growth of CGRP-IR fibers into the disc, which is not innervated normally, and causes pain. ${ }^{18-20}$ In painful scar model of rat plantar, fibrotic thickening of the cutaneous tissues, possible sensitization, and partial degradation of the spinal nerve related to the painful scar were observed. ${ }^{21}$ In addition, upregulation of CGRP in the spinal dorsal horn is led to a decrease in the mechanical pain threshold in the scar of the rat plantar. $^{22}$

It has empirically known that instability may be related to persistent pain in nonunion after fracture. However, there is no previous study about the association of instability with neural changes in nonunion fracture animal model. In this study, the gap induced by $3 \mathrm{~kg}$ load of bending force in the nonunion group tended to be larger than that in the union group. A quantitative assessment of fragment instability by applying bending forces was not performed, however, this result could indicate that increased instability between the bone fragments causes increased CGRP expression in DRG cells and abnormal nerve proliferation in scar tissues. Recent study report that aberrant mechanical stress induces sensory nerve invasion degenerated intervertebral disc in mouse. ${ }^{23}$ The study consists with our results that aberrant instability induced abnormal nerve proliferation.

Clinically, delayed or complete failure of fracture healing remains a problematic complication, and its incidence is between $5 \%$ and $10 \%{ }^{24}$ Our results suggest that early bone healing could minimize the development of persistent pain after fractures. Even if normal fracture healing is not achieved, inhibition of the proliferation of abnormal nerve fibers may decrease the persistent pain.

This study has several limitations. First, CGRP affects not only pain but also bone homeostasis and regeneration. In this study, CGRP was used as a well-established marker of small- and peptide-containing neurons associated with inflammatory pain. In normal rats, half of the neurons innervating the femur bone express CGRP, and osteoarthritis leads to an increase in its expression. ${ }^{25}$ Second, retrograde labeling was not used for quantification of CGRP-IR DRG cells. Therefore, not all CGRP-IR DRG cells counted in this analysis might have innervated the fracture site. However, the femur bone in rats is mainly innervated by DRG neurons at the level of L3. ${ }^{26}$ Third, inflammation due to latex gloves between fracture fragments may have affected the results.
Fourth, in this study, the data was assessed two groups at only single time point. However, at 6 weeks after surgery, callus formation was formed continuously between bone fragments in the union models, while a clear gap was detected between fragments in nonunion models. The time point may be best for verification of our hypothesis. Finally, only types of fracture models were created in this study. However, in preliminary study, we created several models and assessed bone union process. As the results, we used the two models as union and nonunion models to prove our hypothesis. Our nonunion models have can be used for future pain research.

\section{Conclusion}

Nonunion of fractured bone causes increased CGRP expression in DRG cells and persistent nerve proliferation into the scar tissue, which leads to persistent pain. Early achievement of bone union may minimize the neural changes causing persistent pain after fracture.

\section{Acknowledgments}

We are very grateful to Reika Shiraishi for performing histological examinations.

\section{Author Contributions}

All the authors approved the final version of the manuscript. Y.K. had full access to all data in the study and takes responsibility for the integrity of the data and the accuracy of the data analysis. Y.K. designed the experiments, analyzed, and interpreted the results. Y.K. and K. A. wrote the manuscript. Y.K. performed the histological analysis and Reika Shiraishi performed pain-related behavior tests. All authors contributed to data analysis, drafting or revising the article, have agreed on the journal to which the article will be submitted, gave final approval of the version to be published, and agree to be accountable for all aspects of the work.

\section{Disclosure}

Dr Yoshinori Satake reports grants from Eli Lilly Japan K. K., outside the submitted work. The authors report no other conflicts of interest in this work.

\section{References}

1. Kristensen MT. Hip fracture-related pain strongly influences functional performance of patients with an intertrochanteric fracture upon discharge from the hospital. $P M \&$ R. 2013;5:135-141. doi:10.1016/j. pmrj.2012.10.006 
2. Mitchell SAT, Majuta LA, Mantyh PW. New insights in understanding and treating bone fracture pain. Curr Osteoporos Rep. 2018;16:325-332. doi:10.1007/s11914-018-0446-8

3. Alves CJ, Neto E, Sousa DM, et al. Fracture pain-Traveling unknown pathways. Bone. 2016;85:107-114. doi:10.1016/j.bone.2016.01.026

4. Li Z, Meyers CA, Chang L, et al. Fracture repair requires TrkA signaling by skeletal sensory nerves. $J$ Clin Invest. 2019;129:5137-5150. doi:10.1172/JCI128428

5. Alvarez FJ, Morris HR, Priestley JV. Sub-populations of smaller diameter trigeminal primary afferent neurons defined by expression of calcitonin gene-related peptide and the cell surface oligosaccharide recognized by monoclonal antibody LA4. J Neurocytol. 1991;20 (9):716-731. doi:10.1007/BF01187846

6. Snider WD, McMahon SB. Tackling pain at the source: new ideas about nociceptors. Neuron. 1998;20(4):629-632. doi:10.1016/S08966273(00)81003-X

7. Aso K, Ikeuchi M, Izumi M, et al. Nociceptive phenotype of dorsal root ganglia neurons innervating the subchondral bone in rat knee joints. Eur J Pain. 2014;18(2):174-181. doi:10.1002/j.1532-2149.2013.00360.x

8. Castañeda-Corral G, Jimenez-Andrade JM, Bloom AP, et al. The majority of myelinated and unmyelinated sensory nerve fibers that innervate bone express the tropomyosin receptor kinase A. Neuroscience. 2011;178:196-207. doi:10.1016/j.neuroscience.2011.01.039

9. Garcia P, Holstein JH, Maier S, et al. Development of a reliable non-union model in mice. J Surg Res. 2008;147:84-91. doi:10.1016/j.jss.2007.09.013

10. Kokubu T, Hak DJ, Hazelwood SJ, Reddi AH. Development of an atrophic nonunion model and comparison to a closed healing fracture in rat femur. J Orthop Res. 2003;21(3):503-510. doi:10.1016/s07360266(02)00209-7

11. Fujita M, Matsui N, Tsunoda M, Saura R. Establishment of a non-union model using muscle interposition without osteotomy in rats. Kobe J Med Sci. 1998;44:217-233.

12. Nencini S, Ivanusic JJ. The physiology of bone pain. how much do we really know? Front Physiol. 2016;7:157. doi:10.3389/fphys.2016.00157

13. Chartier SR, Thompson ML, Longo G, Fealk MN, Majuta LA, Mantyh PW. Exuberant sprouting of sensory and sympathetic nerve fibers in nonhealed bone fractures and the generation and maintenance of chronic skeletal pain. Pain. 2014;155:2323-2336. doi:10.1016/j.pain.2014.08.026

14. Schneider PS, Sandman E, Martineau PA. Osteoimmunology: effects of standard orthopaedic interventions on inflammatory response and early fracture healing. J Am Acad Orthop Surg. 2018;26:343-352. doi:10.5435/JAAOS-D-16-00646
15. Neagu TP, Țigliş M, Popp CG, Jecan CR. Histological assessment of fracture healing after reduction of the rat femur using two different osteosynthesis methods. Roman $J$ Morphol Embryol. 2016;57:1051-1056.

16. Sun S, Diggins NH, Gunderson ZJ, Fehrenbacher JC, White FA, Kacena MA. No pain, no gain? The effects of pain-promoting neuropeptides and neurotrophins on fracture healing. Bone. 2020;131:115. doi:10.1016/j.bone.2019.115109

17. Mantyh PW. Mechanisms that drive bone pain across the lifespan. $\mathrm{Br}$ J Clin Pharmacol. 2019;85:1103-1113. doi:10.1111/bcp.13801

18. Koshi T, Ohtori S, Inoue $\mathrm{G}$, et al. Lumbar posterolateral fusion inhibits sensory nerve ingrowth into punctured lumbar intervertebral discs and upregulation of CGRP immunoreactive DRG neuron innervating punctured discs in rats. Eur Spine J. 2010;19:593-600. doi:10.1007/s00586-009-1237-9

19. Ohtori S, Orita S, Yamashita M, et al. Existence of a neuropathic pain component in patients with osteoarthritis of the knee. Yonsei Med J. 2012;53:801-805. doi:10.3349/ymj.2012.53.4.801

20. Schaible HG. Mechanisms of chronic pain in osteoarthritis. Curr Rheumatol Rep. 2012;14:549-556. doi:10.1007/s11926-012-0279-x

21. Kajita Y, Suetomi K, Okada T, et al. Behavioral and neuropathological changes in animal models of chronic painful scar. J Orthop Sci. 2013;18(6):1005-1011. doi:10.1007/s00776-013-0453-7

22. Yasui M, Shiraishi Y, Ozaki N, et al. Nerve growth factor and associated nerve sprouting contribute to local mechanical hyperalgesia in a rat model of bone injury. Eur J Pain (London, England). 2012;16:953-965. doi:10.1002/j.1532-2149.2011.00094.x

23. Fu F, Bao R, Yao S, et al. Aberrant spinal mechanical loading stress triggers intervertebral disc degeneration by inducing pyroptosis and nerve ingrowth. Sci Rep. 2021;11(1):772. doi:10.1038/s41598-02080756-6

24. Einhorn TA. Enhancement of fracture-healing. J Bone Joint Surg. 1995;77:940-956. doi:10.2106/00004623-199506000-00016

25. Aso K, Izumi M, Sugimura N, Okanoue Y, Ushida T, Ikeuchi M. Nociceptive phenotype alterations of dorsal root ganglia neurons innervating the subchondral bone in osteoarthritic rat knee joints. Osteoarthritis Cartilage. 2016;24:1596-1603. doi:10.1016/j. joca.2016.04.009

26. Takahashi Y, Chiba T, Kurokawa M, Aoki Y. Dermatomes and the central organization of dermatomes and body surface regions in the spinal cord dorsal horn in rats. J Comp Neurol. 2003;462:29-41. doi:10.1002/cne.10669
Journal of Pain Research

\section{Publish your work in this journal}

The Journal of Pain Research is an international, peer reviewed, open access, online journal that welcomes laboratory and clinical findings in the fields of pain research and the prevention and management of pain Original research, reviews, symposium reports, hypothesis formation and commentaries are all considered for publication. The manuscript

Submit your manuscript here: https://www.dovepress.com/journal-of-pain-research-journa management system is completely online and includes a very quick and fair peer-review system, which is all easy to use. Visit http:// www.dovepress.com/testimonials.php to read real quotes from published authors. 